\title{
Perpetual dual American barrier options for short sellers
}

\author{
Pavel V. Gapeev*
}

\begin{abstract}
We obtain closed-form solutions to the problems of pricing of perpetual American put and call barrier options in the one-dimensional Black-Merton-Scholes model from the point of view of short sellers. The proof is based on the reduction of the original optimal stopping problems for a one-dimensional geometric Brownian motion with positive exponential discounting rates to the equivalent free-boundary problems and the solution of the latter problems by means of the smooth-fit conditions.
\end{abstract}

\section{Introduction}

The main aim of this paper is to present closed-form solutions to the optimal stopping problems of (2.3) for the geometric Brownian motion $S$ defined in (2.1)-(2.2) with positive exponential discounting rates. The process $S$ can describe the price of the underlying risky asset (e.g. a stock) in a model of a financial market. The values of (2.3) are then the rational (or no-arbitrage) prices of perpetual American barrier options in the Black-Merton-Scholes model from the point of view of short sellers (see, e.g. Shiryaev [27; Chapter VIII; Section 2a], Peskir and Shiryaev [22; Chapter VII; Section 25], or Detemple [10], for an extensive overview of other related results in the area).

Optimal stopping problems for one-dimensional diffusion processes with positive exponential discounting rates have been considered in Dynkin [12], Fakeev [13], Mucci [18], Salminen [25], Øksendal and Reikvam [20], and Beibel and Lerche [5]-[6] among others (see also Bensoussan and Lions [7; Theorem 3.19] and Øksendal [19; Chapter X]), for general rewards and infinite time horizon. More recently, such optimal stopping problems were studied in Dayanik and Karatzas [9], Alvarez [1]-[2], Peskir and Shiryaev [22], and Lamberton and Zervos [16] (see the latter references for an extensive discussion). Optimal stopping problems for one-dimensional continuous-time Markov processes with positive exponential discounting rates were recently considered by Shepp and Shiryaev [26], Xia and Zhou [28], Battauz et al. [3]-[4], and De Donno et al. [11] among others. The consideration of positive discounting rates implied the

* London School of Economics, Department of Mathematics, Houghton Street, London WC2A 2AE, United Kingdom; e-mail: p.v.gapeev@lse.ac.uk

Mathematics Subject Classification 2010: Primary 60G40, 60G44, 60J65. Secondary 91B25, 60J60, 35R35.

JEL Classification: G13.

Key words and phrases: Optimal stopping problem, positive discounting rate, Brownian motion, first hitting time, free-boundary problem, instantaneous stopping and smooth fit, a change-of-variable formula with local time on surfaces.

Date: 27 Feb 2019 
appearance of disconnected continuation regions or so-called double continuation regions. In the present paper, we derive explicit expressions for the value functions and stopping boundaries of some optimal stopping problems for one-dimensional geometric Brownian motions with positive exponential discounting rates. It is assumed that the rewards are equal to zero whenever the process reaches certain constant upper or lower levels, so that the value functions are equal to the rational values of perpetual dual American barrier options.

The paper is organised as follows. In Section 2, we introduce the setting and notations of the perpetual dual American up-and-out put and down-and-out call option pricing problems as optimal stopping problems for a geometric Brownian motion with a positive exponential discounting rate and formulate the associated free-boundary problems. In Section 3, we derive closed-form solutions of the latter problems under various relations between the parameters of the model. In Section 4, we verify that the solutions of the free-boundary problems provide the solutions of the original optimal stopping problems. The main results of the paper are stated in Propositions 1 and 2.

\section{Preliminaries}

In this section, we give a formulation of optimal stopping problems with positive exponential discounting rates related to the pricing of perpetual American barrier options from the point of view of short sellers.

\subsection{The model}

For a precise formulation of the problem, let us consider a probability space $(\Omega, \mathcal{F}, P)$ with a standard Brownian motion $B=\left(B_{t}\right)_{t \geq 0}$ and its natural filtration $\left(\mathcal{F}_{t}\right)_{t \geq 0}$. It is further assumed that the filtration $\left(\mathcal{F}_{t}\right)_{t \geq 0}$ is right-continuous and completed by all the sets of $P$-measure zero. Let us define the process $S=\left(S_{t}\right)_{t \geq 0}$ by

$$
S_{t}=s \exp \left(\left(r-\delta-\sigma^{2} / 2\right) t+\sigma B_{t}\right)
$$

which solves the stochastic differential equation

$$
d S_{t}=(r-\delta) S_{t} d t+\sigma S_{t} d B_{t} \quad\left(S_{0}=s\right)
$$

where $s>0$ is fixed, and $r>0, \delta>0$, and $\sigma>0$ are some given constants. It is assumed that the process $S$ describes the price of a risky asset on a financial market, where $r$ is the riskless interest rate of a bank account, $\delta$ is the dividend rate paid to the asset holders, and $\sigma$ is the volatility rate. The purpose of the present paper is to study the optimal stopping problems for the value functions

$$
V_{i}^{*}(s)=\inf _{\tau_{i}} E_{s}\left[e^{r \tau_{i}} G_{i}\left(S_{\tau_{i}}\right) I\left(\tau_{i}<\zeta_{i}\right)\right]
$$

with $G_{1}(s)=K_{1}-s$ and $G_{2}(s)=s-K_{2}$, for some $K_{i}>0$ fixed, where the infima are taken over all stopping times $\tau_{i}, i=1,2$, with respect to the filtration $\left(\mathcal{F}_{t}\right)_{t \geq 0}$. Here $E_{s}$ denotes the expectation with respect to the probability measure $P$ under the assumption that the process 
$S$ starts at $s>0$, and $I(\cdot)$ is the indicator function. We assume that the random times $\zeta_{i}$, $i=1,2$, are given by

$$
\zeta_{1}=\inf \left\{t \geq 0 \mid S_{t} \geq b^{\prime}\right\} \quad \text { and } \quad \zeta_{2}=\inf \left\{t \geq 0 \mid S_{t} \leq a^{\prime}\right\}
$$

for some $0<b^{\prime}<K_{1}$ and $0<K_{2}<a^{\prime}$ fixed. Since the initial probability measure $P$ is a martingale martingale measure (see, e.g. [27; Chapter VII, Section 3g]), the values of (2.3) provide the rational (or no-arbitrage) prices of the perpetual American dual barrier down-andout put and up-and-out call options, respectively. The operations of such contracts can be described as follows. It is assumed that the short sellers receive the fixed payments $V_{i}^{*}(s)$ at time 0 and incur obligations to deliver to the buyers the payoffs $e^{r \tau_{i}} G_{i}\left(S_{\tau_{i}}\right) I\left(\tau_{i}<\zeta_{i}\right)$ at some future times $\tau_{i}, i=1,2$, which the sellers can choose. Observe that when $2 r-\delta \leq 0$ holds, the process $\left(e^{r t} S_{t}\right)_{t \geq 0}$ is a supermartingale closed at zero, so that the optimal exercise time $\tau_{1}^{*}$ is zero, while the optimal exercise time $\tau_{2}^{*}$ coincides with $\zeta_{2}$. In this respect, we further assume that $2 r-\delta>0$ holds.

\subsection{The optimal exercise times}

By means of the results of general theory of optimal stopping (see, e.g. [22; Chapter I, Section 2]), it follows from the structure of the rewards in (2.3) that the optimal stopping times in these problems are given by

$$
\tau_{i}^{*}=\inf \left\{t \geq 0 \mid V_{i}^{*}\left(S_{t}\right)=G_{i}\left(S_{t}\right)\right\}
$$

for every $i=1,2$. We further assume that the optimal stopping times in the problems of (2.3) are of the form

$$
\tau_{1}^{*}=\inf \left\{t \geq 0 \mid S_{t} \notin\left(a_{*}, b^{\prime}\right)\right\} \quad \text { and } \quad \tau_{2}^{*}=\inf \left\{t \geq 0 \mid S_{t} \notin\left(a^{\prime}, b_{*}\right)\right\}
$$

for some numbers $0<a_{*}<b^{\prime}$ and $0<a^{\prime}<b_{*}$ to be determined. By a standard application of Itô's formula (see, e.g. [17; Theorem 4.4]) to the process $e^{r\left(\tau_{i}^{*} \wedge t\right)} G_{i}\left(S_{\tau_{i}^{*} \wedge t}\right)$, we obtain the representations

$$
\begin{aligned}
& e^{r\left(\tau_{i}^{*} \wedge t\right)} G_{i}\left(S_{\tau_{i}^{*} \wedge t}\right) I\left(t<\zeta_{i}\right)=G_{i}(s) \\
& +(-1)^{i} \int_{0}^{\tau_{i}^{*} \wedge t} e^{r u}\left((2 r-\delta) S_{u}-r K_{i}\right) I\left(u<\zeta_{i}\right) d u+N_{t}^{i}
\end{aligned}
$$

for $s<b^{\prime}$ or $s>a^{\prime}$, where the process $\left(N_{\tau_{i}^{*} \wedge t}^{i}\right)_{t \geq 0}$ defined by

$$
N_{\tau_{i}^{*} \wedge t}^{i}=(-1)^{i} \int_{0}^{\tau_{i}^{*} \wedge t} e^{r u} I\left(u<\zeta_{i}\right) \sigma S_{u} d B_{u}
$$

is a continuous square integrable martingale under the probability measure $P_{s}$, for every $i=1,2$. Hence, by applying Doob's optional sampling theorem (see, e.g. [17; Chapter III, Theorem 3.6] or [23; Chapter II, Theorem 3.2]), we obtain that the value functions in (2.3) admit the representations

$$
V_{i}^{*}(s)=G_{i}(s)+(-1)^{i} E_{s}\left[\int_{0}^{\tau_{i}^{*}} e^{r t}\left((2 r-\delta) S_{t}-r K_{i}\right) I\left(t<\zeta_{i}\right) d t\right]
$$


for all $s<b^{\prime}$ or $s>a^{\prime}$, and every $i=1,2$. Thus, it is seen from the structure of the integrand in (2.9) that it is not optimal to exercise the barrier put and call options when $S_{t}>\bar{a}$ with $\bar{a}=r K_{1} /(2 r-\delta)$ and $S_{t}<\underline{b}$ with $\underline{b}=r K_{2} /(2 r-\delta)$, for any $0 \leq t<\tau_{i}^{*} \wedge \zeta_{i}, i=1,2$, respectively. In this respect, we further assume that the optimal stopping boundaries $a_{*}$ and $b_{*}$ in (2.6) should satisfy the inequalities $a_{*}<\bar{a}$ and $b_{*}>\underline{b}$, respectively.

\subsection{The free-boundary problems}

It can be shown by means of Itô's formula that the infinitesimal operator $\mathbb{L}$ of the process $S$ acts on a locally bounded twice continuously differentiable function $F(s)$ on $(0, \infty)$ in the form

$$
(\mathbb{L} F)(s)=(r-\delta) s F^{\prime}(s)+\frac{\sigma^{2} s^{2}}{2} F^{\prime \prime}(s)
$$

for all $s>0$. In order to find closed-form expressions for the unknown value functions $V_{i}^{*}(s)$, $i=1,2$, from (2.3) and the unknown boundaries $a_{*}$ and $b_{*}$ from (2.6), we may use the results of general theory of optimal stopping problems for continuous time Markov processes (see, e.g. [22; Chapter IV, Section 8]) and formulate the associated free-boundary problems

$$
\begin{aligned}
\left(\mathbb{L} V_{i}\right)(s)=-r V_{i}(s) & \text { for } a<s<b^{\prime} \text { or } a^{\prime}<s<b \text { and } i=1,2 \\
\left.V_{1}(s)\right|_{s=a+}=K_{1}-a, & \left.V_{2}(s)\right|_{s=b-}=b-K_{2} \\
\left.V_{1}^{\prime}(s)\right|_{s=a+}=-1, & \left.V_{2}(s)\right|_{s=b-}=1 \\
\left.V_{2}^{\prime}(s)\right|_{s=b^{\prime}-}=0, & \left.V_{2}(s)\right|_{s=a^{\prime}+}=0 \\
V_{1}(s)=K_{1}-s \text { for } s<a, & V_{2}(s)=s-K_{2} \text { for } s>b \\
V_{1}(s)<K_{1}-s \text { for } a<s<b^{\prime}, & V_{2}(s)<s-K_{2} \text { for } a^{\prime}<s<b \\
\left(\mathbb{L} V_{i}\right)(s)>-r V_{i}(s) & \text { for } s<a \text { or } s>b \text { and } i=1,2
\end{aligned}
$$

for some $0<a<b^{\prime}<K_{1}$ and $0<K_{2}<a^{\prime}<b$ to be determined. Observe that the superharmonic characterisation of the value function (see, e.g. [22; Chapter IV, Section 9]) implies that $V_{i}^{*}(s), i=1,2$, are the smallest functions satisfying (2.11)-(2.12) and (2.15)-(2.16) with the boundaries $a_{*}$ and $b_{*}$, respectively.

\section{Solutions to the free-boundary problems}

We now look for functions which solve the free-boundary problems stated in (2.11)-(2.17). For this purpose, we consider three separate cases based on the different relations between the parameters of the model (see Figures 1 and 2 below for computer drawings of the value functions $\left.V_{i}^{*}(s), i=1,2\right)$.

\subsection{The case $0<r<\left(r-\delta-\sigma^{2} / 2\right)^{2} /\left(2 \sigma^{2}\right)$}

Let us first assume that $0<r<\left(r-\delta-\sigma^{2} / 2\right)^{2} /\left(2 \sigma^{2}\right)$ holds. Then, the general solution of the second-order ordinary differential equation in (2.11) has the form

$$
V_{i}(s)=C_{i, 1} s^{\eta_{1}}+C_{i, 2} s^{\eta_{2}}
$$




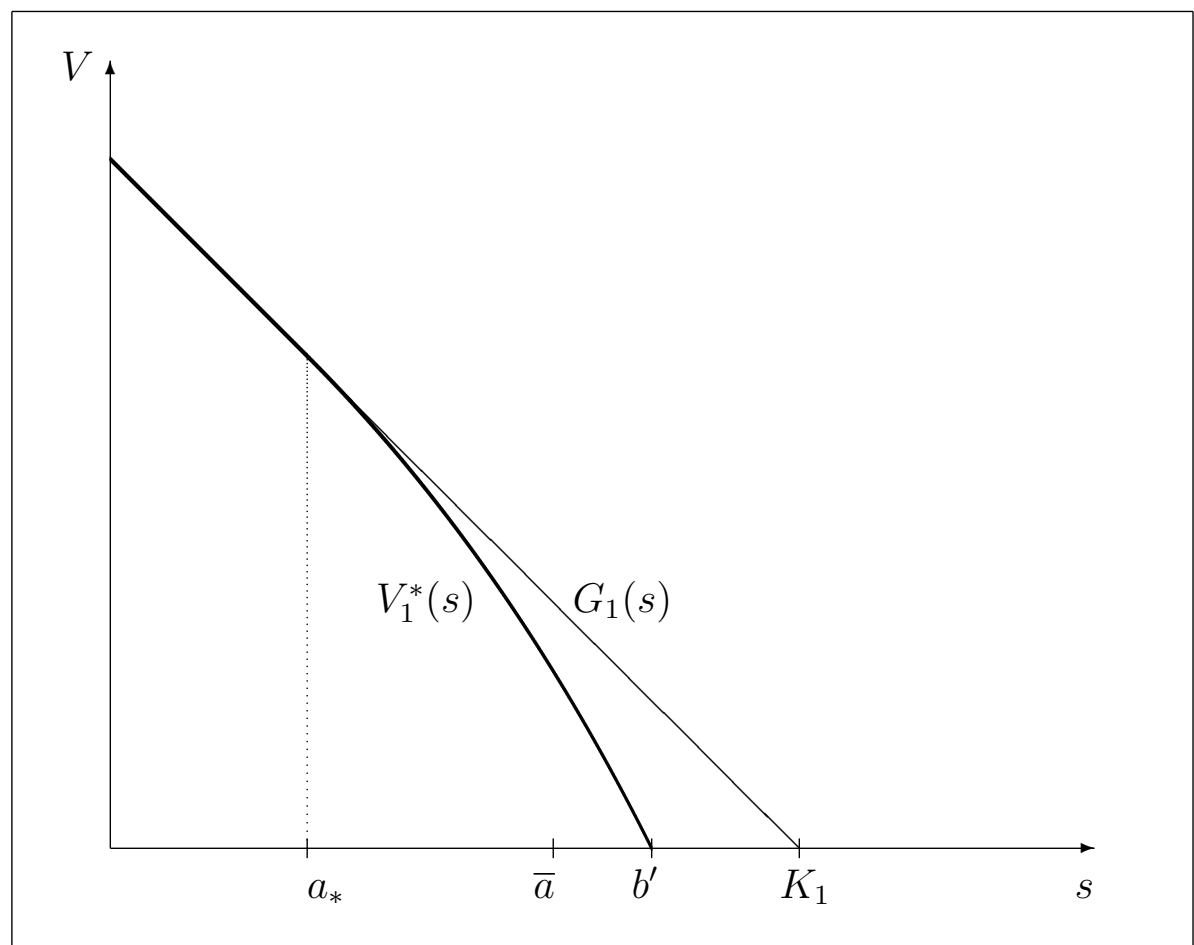

Figure 1. A computer drawing of the value function $V_{1}^{*}(s)$ of the put option.

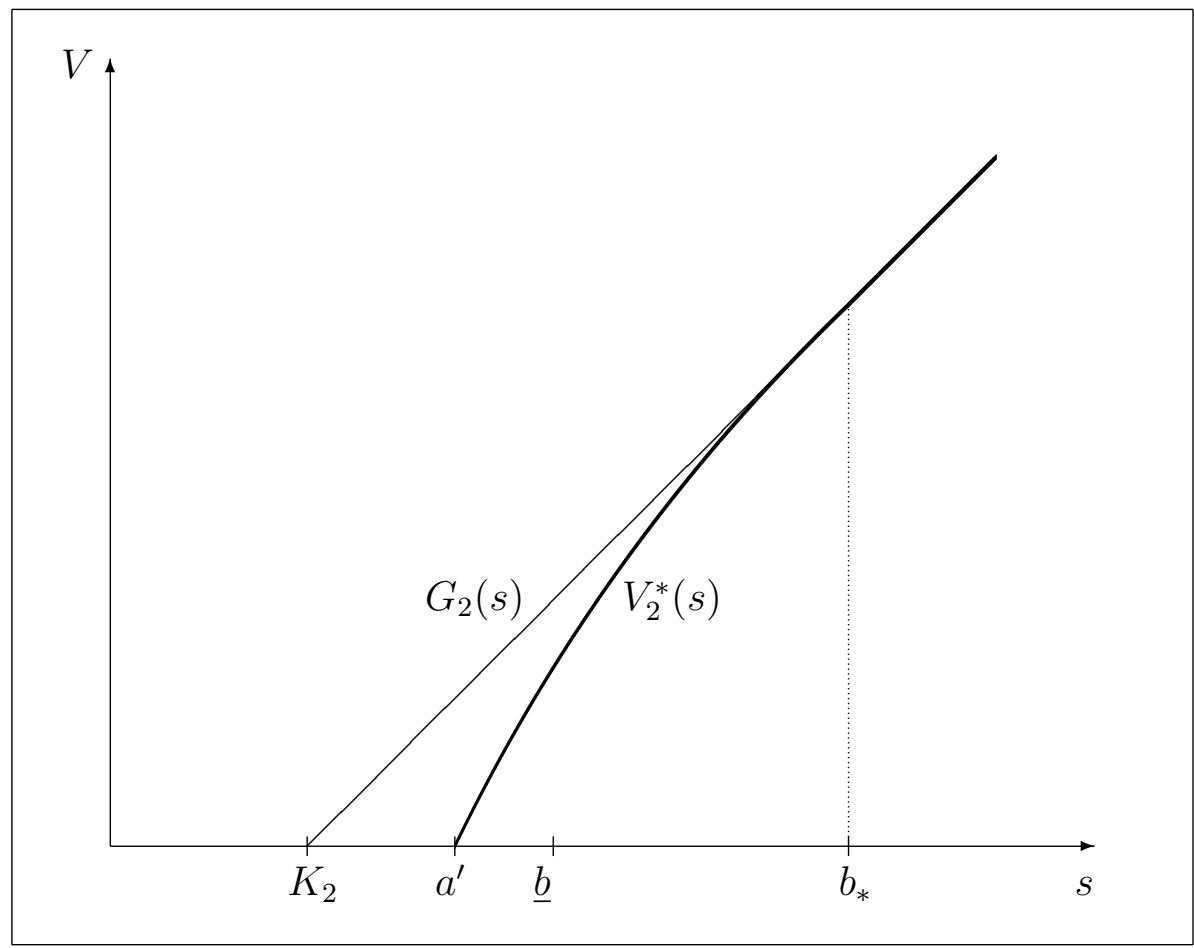

Figure 2. A computer drawing of the value function $V_{2}^{*}(s)$ of the call option. 
where $C_{i, j}, i, j=1,2$, are some arbitrary constants, and $\eta_{j}, j=1,2$, are given by

$$
\eta_{j}=\frac{1}{2}-\frac{r-\delta}{\sigma^{2}}-(-1)^{j} \sqrt{\left(\frac{1}{2}-\frac{r-\delta}{\sigma^{2}}\right)^{2}-\frac{2 r}{\sigma^{2}}}
$$

so that the identity

$$
\frac{\eta_{1}}{\eta_{1}-1} \frac{\eta_{2}}{\eta_{2}-1}=\frac{r}{2 r-\delta}
$$

is satisfied. Note that when $r-\delta<-\sigma^{2} / 2$ holds, we have $1<\eta_{2}<\eta_{1}$, so that $1<\eta_{1} /\left(\eta_{1}-1\right)<$ $\eta_{2} /\left(\eta_{2}-1\right)<r /(2 r-\delta)$ and $\left(\eta_{1}-1\right) /\left(\eta_{2}-1\right)>1$. Moreover, when $-\sigma^{2} / 2 \leq r-\delta<\sigma^{2} / 2$ holds, we have $0<\eta_{2}<\eta_{1}<1$, so that $\eta_{1} /\left(\eta_{1}-1\right)<\eta_{2} /\left(\eta_{2}-1\right)<0$ and $0<\left(\eta_{1}-1\right) /\left(\eta_{2}-1\right)<1$. Finally, when $r-\delta>\sigma^{2} / 2$ holds, we have $\eta_{2}<\eta_{1}<0$, so that $0<r /(2 r-\delta)<\eta_{1} /\left(\eta_{1}-1\right)<$ $\eta_{2} /\left(\eta_{2}-1\right)<1$ and $0<\left(\eta_{1}-1\right) /\left(\eta_{2}-1\right)<1$.

Then, by applying the conditions from (2.12)-(2.14) to the function in (3.1), we get that the equalities

$$
\begin{aligned}
C_{1,1} a^{\eta_{1}}+C_{1,2} a^{\eta_{2}}=K_{1}-a, & C_{2,1} b^{\eta_{1}}+C_{2,2} b^{\eta_{2}}=b-K_{2} \\
C_{1,1} \eta_{1} a^{\eta_{1}}+C_{1,2} \eta_{2} a^{\eta_{2}}=-a, & C_{2,1} \eta_{1} b^{\eta_{1}}+C_{2,2} \eta_{2} b^{\eta_{2}}=b \\
C_{1,1}\left(b^{\prime}\right)^{\eta_{1}}+C_{1,2}\left(b^{\prime}\right)^{\eta_{2}}=0, & C_{2,1}\left(a^{\prime}\right)^{\eta_{1}}+C_{2,2}\left(a^{\prime}\right)^{\eta_{2}}
\end{aligned}
$$

should hold for some $0<a<b^{\prime}<K_{1}$ and $0<K_{2}<a^{\prime}<b$. Hence, solving the systems in (3.4)-(3.6), we obtain that the candidate value function has the form

$$
\begin{aligned}
& V_{1}\left(s ; a_{*}, b^{\prime}\right) \\
& =\frac{1}{\eta_{1}-\eta_{2}}\left(\left(\left(\eta_{2}-1\right) a_{*}-\eta_{2} K_{1}\right)\left(\frac{s}{a_{*}}\right)^{\eta_{1}}+\left(\left(1-\eta_{1}\right) a_{*}+\eta_{1} K_{1}\right)\left(\frac{s}{a_{*}}\right)^{\eta_{2}}\right)
\end{aligned}
$$

for $a_{*}<s<b^{\prime}<K_{1}$, and

$$
\begin{aligned}
& V_{2}\left(s ; b_{*}, a^{\prime}\right) \\
& =\frac{1}{\eta_{1}-\eta_{2}}\left(\left(\left(1-\eta_{2}\right) b_{*}+\eta_{2} K_{2}\right)\left(\frac{s}{b_{*}}\right)^{\eta_{1}}+\left(\left(\eta_{1}-1\right) b_{*}-\eta_{1} K_{2}\right)\left(\frac{s}{b_{*}}\right)^{\eta_{2}}\right)
\end{aligned}
$$

for $K_{2}<a^{\prime}<s<b_{*}$, where $a_{*}$ and $b_{*}$ are determined from the arithmetic equations

$$
\frac{\eta_{1} K_{1}-\left(\eta_{1}-1\right) a}{\eta_{2} K_{1}-\left(\eta_{2}-1\right) a} \equiv \frac{\eta_{1}-1}{\eta_{2}-1}+\frac{\left(\eta_{1}-\eta_{2}\right) K_{1}}{\left(\eta_{2}-1\right)^{2}}\left(a-\frac{\eta_{2} K_{1}}{\eta_{2}-1}\right)^{-1}=\left(\frac{b^{\prime}}{a}\right)^{\eta_{1}-\eta_{2}}
$$

and

$$
\frac{\left(\eta_{1}-1\right) b-\eta_{1} K_{2}}{\left(\eta_{2}-1\right) b-\eta_{2} K_{2}} \equiv \frac{\eta_{1}-1}{\eta_{2}-1}+\frac{\left(\eta_{1}-\eta_{2}\right) K_{2}}{\left(\eta_{2}-1\right)^{2}}\left(b-\frac{\eta_{2} K_{2}}{\eta_{2}-1}\right)^{-1}=\left(\frac{a^{\prime}}{b}\right)^{\eta_{1}-\eta_{2}}
$$

respectively.

In order to consider the put option case, we observe from the mentioned above properties of the numbers $\eta_{j}, j=1,2$, from (3.2) and the identity in (3.3) that, if $r-\delta<-\sigma^{2} / 2$ holds, then the equation in (3.9) has a unique solution $a_{*}$ on the interval $\left(0, b^{\prime}\right)$ such that $a_{*}<\bar{a}$ with 
$\bar{a}=r K_{1} /(2 r-\delta)$. Then, if $-\sigma^{2} / 2 \leq r-\delta<\sigma^{2} / 2$ holds, then the equation in (3.9) has a unique solution $a_{*}$ on the interval $\left(0, b^{\prime}\right)$ such that $a_{*}<\bar{a}$, whenever either the inequality $r \leq \delta$ or the inequalities $r>\delta$ and $b^{\prime} \leq \bar{a}$ are satisfied. Finally, if $r-\delta>\sigma^{2} / 2$ holds, then the equation in (3.9) has no solution $a_{*}$ on the interval $\left(0, b^{\prime}\right)$ such that $a_{*}<\bar{a}$, and thus, we can set $a_{*}=0$, so that $V_{1}\left(s ; a_{*}, b^{\prime}\right)=0$, for all $0<s<b^{\prime}$.

In order to consider the call option case, we observe from the mentioned properties of $\eta_{j}$, $j=1,2$, and the identity above that, if $r-\delta>\sigma^{2} / 2$ holds, then the equation in (3.10) has a unique solution $b_{*}$ on the interval $\left(a^{\prime}, \infty\right)$ such that $b_{*}>\underline{b}$ with $\underline{b}=r K_{2} /(2 r-\delta)$. Then, if $-\sigma^{2} / 2 \leq r-\delta<\sigma^{2} / 2$ holds, then the equation in (3.10) has a unique solution $b_{*}$ on the interval $\left(a^{\prime}, \infty\right)$ such that $b_{*}>\underline{b}$, whenever either the inequality $r \geq \delta$ or the inequalities $r<\delta$ and $a^{\prime} \geq \underline{b}$ are satisfied. Finally, if $r-\delta<-\sigma^{2} / 2$ holds, then the equation in (3.10) has no solution $b_{*}$ on the interval $\left(a^{\prime}, \infty\right)$ such that $b_{*}>\underline{b}$, and thus, we can set $b_{*}=\infty$, so that $V_{2}\left(s ; b_{*}, a^{\prime}\right)=0$, for all $s>a^{\prime}$.

\subsection{The case $r=\left(r-\delta-\sigma^{2} / 2\right)^{2} /\left(2 \sigma^{2}\right)$}

Let us now assume that $r=\left(r-\delta-\sigma^{2} / 2\right)^{2} /\left(2 \sigma^{2}\right)$ holds. Then, the general solution of the ordinary differential equation in (2.11) has the form

$$
V_{i}(s)=C_{i, 1} s^{\lambda} \ln s+C_{i, 2} s^{\lambda}
$$

where $C_{i, j}, i, j=1,2$, are some arbitrary constants, and $\lambda$ is given by:

$$
\lambda=\frac{1}{2}-\frac{r-\delta}{\sigma^{2}}
$$

so that the identity

$$
\left(\frac{\lambda}{\lambda-1}\right)^{2}=\frac{r}{2 r-\delta}
$$

is satisfied. Observe that the value of $\lambda$ in (3.12) coincides with the values of $\eta_{i}, i=1,2$, under the current assumption $r=\left(r-\delta-\sigma^{2} / 2\right)^{2} /\left(2 \sigma^{2}\right)$, since the appropriate expression under the root sign becomes zero in this case. We also note that when $r-\delta<-\sigma^{2} / 2$ holds, we have $\lambda>1$, so that $\lambda /(\lambda-1)>1$ and $1 /(\lambda-1)>0$. Moreover, when $-\sigma^{2} / 2 \leq r-\delta<\sigma^{2} / 2$ holds, we have $0<\lambda<1$, so that $\lambda /(\lambda-1)<0$ and $1 /(\lambda-1)<0$. Finally, when $r-\delta>\sigma^{2} / 2$ holds, we have $\lambda<0$, so that $0<\lambda /(\lambda-1)<1$ and $1 /(\lambda-1)<0$.

Then, by applying the conditions from (2.12)-(2.14) to the function in (3.11), we get that the equalities

$$
\begin{array}{cl}
C_{1,1} a^{\lambda} \ln a+C_{1,2} a^{\lambda}=K_{1}-a, & C_{2,1} b^{\lambda} \ln b+C_{2,2} b^{\lambda}=b-K_{2} \\
C_{1,1} a^{\lambda}(\lambda \ln a+1)+C_{1,2} \lambda a^{\lambda}=-a, & C_{2,1} b^{\lambda}(\lambda \ln b+1)+C_{2,2} \lambda b^{\lambda}=b \\
C_{1,1}\left(b^{\prime}\right)^{\lambda} \ln b^{\prime}+C_{1,2}\left(b^{\prime}\right)^{\lambda}=0, & C_{2,1}\left(a^{\prime}\right)^{\lambda} \ln a^{\prime}+C_{2,2}\left(a^{\prime}\right)^{\lambda}=0
\end{array}
$$

should hold for some $0<a<b^{\prime}<K_{1}$ and $0<K_{2}<a^{\prime}<b$. Thus, solving the systems in (3.14)-(3.16), we obtain that the candidate value function has the form

$$
V_{1}\left(s ; a_{*}, b^{\prime}\right)=\left((\lambda-1) a_{*}-\lambda K_{1}\right)\left(\frac{s}{a_{*}}\right)^{\lambda} \ln \left(\frac{s}{a_{*}}\right)+\left(K_{1}-a_{*}\right)\left(\frac{s}{a_{*}}\right)^{\lambda}
$$


for $a_{*}<s<b^{\prime}<K_{1}$, and

$$
V_{2}\left(s ; b_{*}, a^{\prime}\right)=\left(\lambda K_{2}-(\lambda-1) b_{*}\right)\left(\frac{s}{b_{*}}\right)^{\lambda} \ln \left(\frac{s}{b_{*}}\right)+\left(b_{*}-K_{2}\right)\left(\frac{s}{b_{*}}\right)^{\lambda}
$$

for $K_{2}<a^{\prime}<s<b_{*}$, where $a_{*}$ and $b_{*}$ are determined from the arithmetic equations

$$
\frac{K_{1}-a}{\lambda K_{1}-(\lambda-1) a} \equiv \frac{1}{\lambda-1}+\frac{K_{1}}{(\lambda-1)^{2}}\left(a-\frac{\lambda K_{1}}{\lambda-1}\right)^{-1}=\ln \left(\frac{b^{\prime}}{a}\right)
$$

and

$$
\frac{b-K_{2}}{(\lambda-1) b-\lambda K_{2}} \equiv \frac{1}{\lambda-1}+\frac{K_{2}}{(\lambda-1)^{2}}\left(b-\frac{\lambda K_{2}}{\lambda-1}\right)^{-1}=\ln \left(\frac{a^{\prime}}{b}\right)
$$

respectively.

In order to consider the put option case, we observe from the expressions for $\lambda$ in (3.12) and (3.13) that, if $r-\delta<-\sigma^{2} / 2$ holds, then the equation in (3.19) has a unique solution $a_{*}$ on the interval $\left(0, b^{\prime}\right)$ such that $a_{*}<\bar{a}$ with $\bar{a}=r K_{1} /(2 r-\delta)$. Then, if $-\sigma^{2} / 2 \leq r-\delta<\sigma^{2} / 2$ holds, then the equation in (3.19) has a unique solution $a_{*}$ on the interval $\left(0, b^{\prime}\right)$ such that $a_{*}<\bar{a}$, whenever either the inequality $r \leq \delta$ or the inequalities $r>\delta$ and $b^{\prime} \leq \bar{a}$ are satisfied. Finally, if $r-\delta>\sigma^{2} / 2$ holds, then the equation in (3.19) has no solution $a_{*}$ on the interval $\left(0, b^{\prime}\right)$ such that $a_{*}<\bar{a}$, and thus, we can set $a_{*}=0$, so that $V_{1}\left(s ; a_{*}, b^{\prime}\right)=0$, for all $0<s<b^{\prime}$.

In order to consider the call option case, we observe from the mentioned above properties of $\lambda$ that, if $r-\delta>\sigma^{2} / 2$ holds, then the equation in (3.20) has a unique solution $b_{*}$ on the interval $\left(a^{\prime}, \infty\right)$ such that $b_{*}>\underline{b}$ with $\underline{b}=r K_{2} /(2 r-\delta)$. Then, if $-\sigma^{2} / 2 \leq r-\delta<\sigma^{2} / 2$ holds, then the equation in (3.20) has a unique solution $b_{*}$ on the interval $\left(a^{\prime}, \infty\right)$ such that $b_{*}>\underline{b}$, whenever either the inequality $r \geq \delta$ or the inequalities $r<\delta$ and $a^{\prime} \geq \underline{b}$ are satisfied. Finally, if $r-\delta<-\sigma^{2} / 2$ holds, then the equation in (3.20) has no solution $b_{*}$ on the interval $\left(a^{\prime}, \infty\right)$ such that $b_{*}>\underline{b}$, and thus, we can set $b_{*}=\infty$, so that $V_{2}\left(s ; b_{*}, a^{\prime}\right)=0$, for all $s>a^{\prime}$.

\subsection{The case $r>\left(r-\delta-\sigma^{2} / 2\right)^{2} /\left(2 \sigma^{2}\right)$}

Let us finally assume that $r>\left(r-\delta-\sigma^{2} / 2\right)^{2} /\left(2 \sigma^{2}\right)$ holds. Then, the general solution of the ordinary differential equation in (2.11) has the form

$$
V_{i}(s)=C_{i, 1} s^{\lambda} \sin (\theta \ln s)+C_{i, 2} s^{\lambda} \cos (\theta \ln s)
$$

where $C_{i, j}, i, j=1,2$, are some arbitrary constants, while $\lambda$ is given by (3.12) and $\theta$ is set as

$$
\theta=\sqrt{\frac{2 r}{\sigma^{2}}-\left(\frac{1}{2}-\frac{r-\delta}{\sigma^{2}}\right)^{2}}
$$


Hence, by applying the conditions from (2.12)-(2.14) to the function in (3.21), we get that the equalities

$$
\begin{aligned}
& C_{1,1} a^{\lambda} \sin (\theta \ln a)+C_{1,2} a^{\lambda} \cos (\theta \ln a)=K_{1}-a, \\
& C_{2,1} b^{\lambda} \sin (\theta \ln b)+C_{2,2} b^{\lambda} \cos (\theta \ln b)=b-K_{2} \\
& \left(C_{1,1} \lambda-C_{1,2} \theta\right) a^{\lambda} \sin (\theta \ln a)+\left(C_{1,1} \theta+C_{1,2} \lambda\right) a^{\lambda} \cos (\theta \ln a)=-a, \\
& \left(C_{2,1} \lambda-C_{2,2} \theta\right) a^{\lambda} \sin (\theta \ln a)+\left(C_{2,1} \theta+C_{2,2} \lambda\right) a^{\lambda} \cos (\theta \ln a)=b \\
& C_{1,1}\left(b^{\prime}\right)^{\lambda} \sin \left(\theta \ln b^{\prime}\right)+C_{1,2}\left(b^{\prime}\right)^{\lambda} \cos \left(\theta \ln b^{\prime}\right)=0 \\
& C_{2,1}\left(a^{\prime}\right)^{\lambda} \sin \left(\theta \ln a^{\prime}\right)+C_{2,2}\left(a^{\prime}\right)^{\lambda} \cos \left(\theta \ln a^{\prime}\right)=0
\end{aligned}
$$

should hold for some $0<a<b^{\prime}<K_{1}$ and $0<K_{2}<a^{\prime}<b$. Thus, solving the systems in (3.23)-(3.28), we obtain that the candidate value function has the form

$$
\begin{aligned}
& V_{1}\left(s ; a_{*}, b^{\prime}\right) \\
& =\left((\lambda-1) a_{*}-\lambda K_{1}\right)\left(\frac{s}{a_{*}}\right)^{\lambda} \sin \left(\theta \ln \left(\frac{s}{a_{*}}\right)\right)+\theta\left(K_{1}-a_{*}\right)\left(\frac{s}{a_{*}}\right)^{\lambda} \cos \left(\theta \ln \left(\frac{s}{a_{*}}\right)\right)
\end{aligned}
$$

for $a_{*}<s<b^{\prime}<K_{1}$, and

$$
\begin{aligned}
& V_{2}\left(s ; b_{*}, a^{\prime}\right) \\
& =\left(\lambda K_{2}-(\lambda-1) b_{*}\right)\left(\frac{s}{b_{*}}\right)^{\lambda} \sin \left(\theta \ln \left(\frac{s}{b_{*}}\right)\right)+\theta\left(b_{*}-K_{2}\right)\left(\frac{s}{b_{*}}\right)^{\lambda} \cos \left(\theta \ln \left(\frac{s}{b_{*}}\right)\right)
\end{aligned}
$$

for $K_{2}<a^{\prime}<s<b_{*}$, where $a_{*}$ and $b_{*}$ are determined from the arithmetic equations

$$
\arctan \left(\frac{\theta}{\lambda-1}+\frac{\theta K_{1}}{(\lambda-1)^{2}}\left(a-\frac{\lambda K_{1}}{\lambda-1}\right)^{-1}\right)=\theta \ln \left(\frac{b^{\prime}}{a}\right)
$$

and

$$
\arctan \left(\frac{\theta}{\lambda-1}+\frac{\theta K_{2}}{(\lambda-1)^{2}}\left(b-\frac{\lambda K_{2}}{\lambda-1}\right)^{-1}\right)=\theta \ln \left(\frac{a^{\prime}}{b}\right)
$$

respectively.

In order to consider the put option case, we observe from the expressions for $\lambda$ in (3.12) and (3.13) that, if $r-\delta<-\sigma^{2} / 2$ holds, then the equation in (3.31) has a unique solution $a_{*}$ on the interval $\left(0, b^{\prime}\right)$ such that $a_{*}<\bar{a}$ with $\bar{a}=r K_{1} /(2 r-\delta)$. Then, if $-\sigma^{2} / 2 \leq r-\delta<\sigma^{2} / 2$ holds, then the equation in (3.31) has a unique solution $a_{*}$ on the interval $\left(0, b^{\prime}\right)$ such that $a_{*}<\bar{a}$, whenever either the inequality $r \leq \delta$ or the inequalities $r>\delta$ and $b^{\prime} \leq \bar{a}$ are satisfied. Finally, if $r-\delta>\sigma^{2} / 2$ holds, then the equation in (3.31) has no solution $a_{*}$ on the interval $\left(0, b^{\prime}\right)$ such that $a_{*}<\bar{a}$, and thus, we can set $a_{*}=0$, so that $V_{1}\left(s ; a_{*}, b^{\prime}\right)=0$, for all $0<s<b^{\prime}$.

In order to consider the call option case, we observe from the mentioned above properties of $\lambda$ that, if $r-\delta>\sigma^{2} / 2$ holds, then the equation in (3.32) has a unique solution $b_{*}$ on the interval $\left(a^{\prime}, \infty\right)$ such that $b_{*}>\underline{b}$ with $\underline{b}=r K_{2} /(2 r-\delta)$. Then, if $-\sigma^{2} / 2 \leq r-\delta<\sigma^{2} / 2$ holds, then the equation in (3.32) has a unique solution $b_{*}$ on the interval $\left(a^{\prime}, \infty\right)$ such that $b_{*}>\underline{b}$, whenever either the inequality $r \geq \delta$ or the inequalities $r<\delta$ and $a^{\prime} \geq \underline{b}$ are satisfied. Finally, if $r-\delta<-\sigma^{2} / 2$ holds, then the equation in (3.32) has no solution $b_{*}$ on the interval $\left(a^{\prime}, \infty\right)$ such that $b_{*}>\underline{b}$, and thus, we can set $b_{*}=\infty$, so that $V_{2}\left(s ; b_{*}, a^{\prime}\right)=0$, for all $s>a^{\prime}$. 


\section{Main results}

In this section, we show that the solutions of the free-boundary problems from the previous section provides the solutions of the initial optimal stopping problems of (2.3).

Proposition 4.1 Let the process $S$ be given by (2.1), with some $r>0, \delta>0$, and $\sigma>0$ fixed, and such that $2 r-\delta>0$. Then, the value function of the perpetual American dual barrier (up-and-out) put option in (2.3) has the form

$$
V_{1}^{*}(s)= \begin{cases}V_{1}\left(s ; a_{*}, b^{\prime}\right), & \text { if } a_{*}<s<b^{\prime} \\ K_{1}-s, & \text { if } s \leq a_{*}\end{cases}
$$

for some $0<b^{\prime}<K_{1}$ fixed, and $\tau_{1}^{*}$ from (2.6) is an optimal stopping time, where we have the following assertions:

(i) When $0<r<\left(r-\delta-\sigma^{2} / 2\right)^{2} /\left(2 \sigma^{2}\right)$ holds, the function $V_{1}\left(s ; a_{*}, b^{\prime}\right)$ takes the expression of (3.7), while if $r-\delta<-\sigma^{2} / 2$ then the equation in (3.9) has a unique solution $a_{*}$ on the interval $\left(0, b^{\prime} \wedge \bar{a}\right)$ with $\bar{a}=r K_{1} /(2 r-\delta)$, if $-\sigma^{2} / 2 \leq r-\delta<\sigma^{2} / 2$ and either the inequality $r \leq \delta$ or the inequalities $r>\delta$ and $b^{\prime} \leq \bar{a}$ are satisfied then the equation in (3.9) has a unique solution $a_{*}$ on the interval $\left(0, b^{\prime} \wedge \bar{a}\right)$, as well as if $r-\delta>\sigma^{2} / 2$ then the equation in (3.9) has no solution $a_{*}$ on the interval $\left(0, b^{\prime} \wedge \bar{a}\right)$, so that $a_{*}=0$ and $V_{1}\left(s ; a_{*}, b^{\prime}\right) \equiv 0$.

(ii) When $r=\left(r-\delta-\sigma^{2} / 2\right)^{2} /\left(2 \sigma^{2}\right)$ holds, the function $V_{1}\left(s ; a_{*}, b^{\prime}\right)$ takes the expression of (3.17), while if $r-\delta<-\sigma^{2} / 2$ then the equation in (3.19) has a unique solution $a_{*}$ on the interval $\left(0, b^{\prime} \wedge \bar{a}\right)$, if $-\sigma^{2} / 2 \leq r-\delta<\sigma^{2} / 2$ and either the inequality $r \leq \delta$ or the inequalities $r>\delta$ and $b^{\prime} \leq \bar{a}$ are satisfied then the equation in (3.19) has a unique solution $a_{*}$ on the interval $\left(0, b^{\prime} \wedge \bar{a}\right)$, as well as if $r-\delta>\sigma^{2} / 2$ then the equation in (3.19) has no solution $a_{*}$ on the interval $\left(0, b^{\prime} \wedge \bar{a}\right)$, so that $a_{*}=0$ and $V_{1}\left(s ; a_{*}, b^{\prime}\right) \equiv 0$.

(iii) When $r>\left(r-\delta-\sigma^{2} / 2\right)^{2} /\left(2 \sigma^{2}\right)$ holds, the function $V_{1}\left(s ; a_{*}, b^{\prime}\right)$ takes the expression of (3.29), while if $r-\delta<-\sigma^{2} / 2$ then the equation in (3.31) has a unique solution $a_{*}$ on the interval $\left(0, b^{\prime} \wedge \bar{a}\right)$, if $-\sigma^{2} / 2 \leq r-\delta<\sigma^{2} / 2$ and either the inequality $r \leq \delta$ or the inequalities $r>\delta$ and $b^{\prime} \leq \bar{a}$ are satisfied then the equation in (3.31) has a unique solution $a_{*}$ on the interval $\left(0, b^{\prime} \wedge \bar{a}\right)$, as well as if $r-\delta>\sigma^{2} / 2$ then the equation in (3.31) has no solution $a_{*}$ on the interval $\left(0, b^{\prime} \wedge \bar{a}\right)$, so that $a_{*}=0$ and $V_{1}\left(s ; a_{*}, b^{\prime}\right) \equiv 0$.

Proposition 4.2 Let the process $S$ be given by (2.1), with some $r>0, \delta>0$, and $\sigma>0$ fixed, and such that $2 r-\delta>0$. Then, the value function of the perpetual American dual barrier (down-and-out) call option in (2.3) has the form:

$$
V_{2}^{*}(s)= \begin{cases}V_{2}\left(s ; b_{*}, a^{\prime}\right), & \text { if } a^{\prime}<s<b_{*} \\ s-K_{2}, & \text { if } s \geq b_{*}\end{cases}
$$

for some $0<K_{2}<a^{\prime}$ fixed, and $\tau_{2}^{*}$ from (2.6) is an optimal stopping time, where we have the following assertions:

(i) When $0<r<\left(r-\delta-\sigma^{2} / 2\right)^{2} /\left(2 \sigma^{2}\right)$ holds, the function $V_{2}\left(s ; b_{*}, a^{\prime}\right)$ takes the expression of (3.8), while if $r-\delta>\sigma^{2} / 2$ then the equation in (3.10) has a unique solution $b_{*}$ on the interval $\left(a^{\prime} \vee \underline{b}, \infty\right)$ with $\underline{b}=r K_{2} /(2 r-\delta)$, if $-\sigma^{2} / 2 \leq r-\delta<\sigma^{2} / 2$ and either the inequality $r \geq \delta$ or the inequalities $r<\delta$ and $a^{\prime} \geq \underline{b}$ are satisfied then the equation in (3.10) has a unique solution 
$b_{*}$ on the interval $\left(a^{\prime} \vee \underline{b}, \infty\right)$, while if $r-\delta<-\sigma^{2} / 2$ then the equation in (3.10) has no solution $b_{*}$ on the interval $\left(a^{\prime} \vee \underline{b}, \infty\right)$, so that $b_{*}=\infty$ and $V_{2}\left(s ; b_{*}, a^{\prime}\right) \equiv 0$.

(ii) When $r=\left(r-\bar{\delta}-\sigma^{2} / 2\right)^{2} /\left(2 \sigma^{2}\right)$ holds, the function $V_{2}\left(s ; b_{*}, a^{\prime}\right)$ takes the expression of (3.18), while if $r-\delta>\sigma^{2} / 2$ then the equation in (3.20) has a unique solution $b_{*}$ on the interval $\left(a^{\prime} \vee \underline{b}, \infty\right)$, if $-\sigma^{2} / 2 \leq r-\delta<\sigma^{2} / 2$ and either the inequality $r \geq \delta$ or the inequalities $r<\delta$ and $a^{\prime} \geq \underline{b}$ are satisfied then the equation in (3.20) has a unique solution $b_{*}$ on the interval $\left(a^{\prime} \vee \underline{b}, \infty\right)$, as well as if $r-\delta<-\sigma^{2} / 2$ then the equation in (3.20) has no solution $b_{*}$ on the interval $\left(a^{\prime} \vee \underline{b}, \infty\right)$, so that $b_{*}=\infty$ and $V_{2}\left(s ; b_{*}, a^{\prime}\right) \equiv 0$.

(iii) When $r>\left(r-\delta-\sigma^{2} / 2\right)^{2} /\left(2 \sigma^{2}\right)$ holds, the function $V_{2}\left(s ; b_{*}, a^{\prime}\right)$ takes the expression of (3.30), while if $r-\delta>\sigma^{2} / 2$ then the equation in (3.32) has a unique solution $b_{*}$ on the interval $\left(a^{\prime} \vee \underline{b}, \infty\right)$, if $-\sigma^{2} / 2 \leq r-\delta<\sigma^{2} / 2$ and either the inequality $r \geq \delta$ or the inequalities $r<\delta$ and $a^{\prime} \geq \underline{b}$ are satisfied then the equation in (3.32) has a unique solution $b_{*}$ on the interval $\left(a^{\prime} \vee \underline{b}, \infty\right)$, as well as if $r-\delta<-\sigma^{2} / 2$ then the equation in (3.32) has no solution $b_{*}$ on the interval $\left(a^{\prime} \vee \underline{b}, \infty\right)$, so that $b_{*}=\infty$ and $V_{2}\left(s ; b_{*}, a^{\prime}\right) \equiv 0$.

Proof: In order to verify the assertions stated above, we are left to show that the functions introduced in (4.1) and (4.2) coincide with the value functions in (2.3), and that the stopping times $\tau_{i}^{*}, i=1,2$, in (2.6) are optimal with the boundaries $a_{*}$ and $b_{*}$ specified above. For this purpose, let us denote by $V_{i}(s), i=1,2$, the right-hand sides of the expressions in (4.1) and (4.2). Then, we may conclude from the equations in (2.11) that the derivatives $V_{i}^{\prime}(s)$, $i=1,2$, are continuously differentiable on $\left(a_{*}, b^{\prime}\right)$ and $\left(a^{\prime}, b_{*}\right)$, respectively. Hence, according to the conditions of (2.12)-(2.15), applying the change-of-variable formula from [21] (see also [22; Chapter II, Section 3.5] for a summary of the related results on the local time-space formula as well as further references), we get

$$
e^{r t} V_{i}\left(S_{t}\right)=V_{i}(s)+\int_{0}^{t} e^{r u}\left(\mathbb{L} V_{i}+r V_{i}\right)\left(S_{u}\right) I\left(S_{u} \neq a_{*} \text { or } S_{u} \neq b_{*}\right) d u+M_{t}^{i}
$$

for all $t \geq 0$, where the processes $M^{i}=\left(M_{t}^{i}\right)_{t \geq 0}, i=1,2$, defined by:

$$
M_{t}^{i}=\int_{0}^{t} e^{r u} V_{i}^{\prime}\left(S_{u}\right) \sigma S_{u} d B_{u}
$$

are continuous local martingales with respect to the probability measure $P_{s}$. Observe that the time spent by $S$ at the points $a_{*}$ and $b_{*}$ is of Lebesgue measure zero, and thus, the indicator which appear in the integral of (4.3) can be ignored (see, e.g. [8; Chapter II, Section 1]).

By using straightforward calculations and the arguments from the previous section, it is verified that the inequalities $\left(\mathbb{L} V_{i}+r V_{i}\right)(s) \geq 0, i=1,2$, hold, for all $s<b^{\prime}$ such that $s \neq a_{*}$ or $s>a^{\prime}$ such that $s \neq b_{*}$, respectively. Moreover, it is shown by means of standard arguments that the inequalities in (2.16) hold, which together with the conditions of (2.12)-(2.15) imply that $V_{i}(s) \leq G_{i}(s), i=1,2$, holds, for all $s<b^{\prime}$ or $s>a^{\prime}$, respectively. Hence, the expression in (4.3) yields that the inequalities

$$
e^{r \tau_{i}} G_{i}\left(S_{\tau_{i}}\right) \geq e^{r \tau_{i}} V_{i}\left(S_{\tau_{i}}\right) \geq V_{i}(s)+M_{\tau_{i}}^{i}
$$

hold for any stopping times $\tau_{i}, i=1,2$, of the process $S$ started at $s>0$. Let $\left(\varkappa_{i}^{n}\right)_{n \in \mathbb{N}}$ be arbitrary localising sequences of stopping times for the processes $M^{i}, i=1,2$, respectively. 
Taking in (4.5) the expectation with respect to the measure $P_{s}$, by means of the optional sampling theorem (see, e.g. [15; Chapter I, Theorem 3.22]), we get that the inequalities

$$
\begin{aligned}
E_{s}\left[e^{r\left(\tau_{i} \wedge \varkappa_{i}^{n}\right)} G_{i}\left(S_{\tau_{i} \wedge \varkappa_{i}^{n}}\right)\right] & \geq E_{s}\left[e^{r\left(\tau_{i} \wedge \varkappa_{i}^{n}\right)} V_{i}\left(S_{\tau_{i} \wedge \varkappa_{i}^{n}}\right)\right] \\
& \geq V_{i}(s)+E_{s}\left[M_{\tau_{i} \wedge \varkappa_{i}^{n}}^{i}\right]=V_{i}(s)
\end{aligned}
$$

hold, for all $s>0$ and every $i=1,2$. Hence, letting $n$ go to infinity and using Fatou's lemma, we obtain

$$
E_{s}\left[e^{r \tau_{i}} G\left(S_{\tau_{i}}\right)\right] \geq E_{s}\left[e^{r \tau_{i}} V\left(S_{\tau_{i}}\right)\right] \geq V_{i}(s)
$$

for any stopping times $\tau_{i}, i=1,2$, and all $s>0$. By virtue of the structure of the stopping times in (2.6), it is readily seen that the equalities in (4.7) hold with $\tau_{i}^{*}$ instead of $\tau_{i}, i=1,2$, when either $s \leq a_{*}$ or $s \geq b_{*}$.

It remains us to show that the equalities are attained in (4.7) when $\tau_{i}^{*}$ replace $\tau_{i}, i=1$, 2 , for $a_{*}<s<b^{\prime}$ or $a^{\prime}<s<b_{*}$, respectively. By virtue of the fact that the functions $V_{1}\left(s ; a_{*}, b^{\prime}\right)$ and $V_{2}\left(s ; b_{*}, a^{\prime}\right)$ and the boundaries $a_{*}$ and $b_{*}$ satisfy the conditions in (2.11) and (2.12), it follows from the expression in (4.3) and the structure of the stopping times in (2.6) that the equalities

$$
e^{r\left(\tau_{i}^{*} \wedge \varkappa_{i}^{n}\right)} V_{i}\left(S_{\tau_{i}^{*} \wedge \varkappa_{i}^{n}}\right)=V_{i}(s)+M_{\tau_{i}^{*} \wedge \varkappa_{i}^{n}}^{i}
$$

are satisfied, for all $a_{*}<s<b^{\prime}$ or $a^{\prime}<s<b_{*}$, and any localising sequence $\left(\varkappa_{i}^{n}\right)_{n \in \mathbb{N}}$ of $M^{i}$, $i=1,2$. Observe that the form of the gain functions $G_{i}(s)$ together with the explicit expressions for the candidate value functions in (3.7)-(3.8), (3.17)-(3.18), and (3.29)-(3.30) yield that the conditions

$$
E_{S}\left[\sup _{t \geq 0} e^{r\left(\tau_{i}^{*} \wedge t\right)} V_{i}\left(S_{\tau_{i}^{*} \wedge t}\right)\right]<\infty
$$

hold, for all $a_{*}<s<b^{\prime}$ and $a^{\prime}<s<b_{*}$, as well as the variables $e^{r \tau^{*}} V_{i}\left(S_{\tau_{i}^{*}}\right)$ are bounded on the events $\left\{\tau_{i}^{*}=\infty\right\}, i=1,2$ ( $P_{s^{-}}$-a.s. $)$. Hence, taking into account the property in (4.9), we conclude from the expression in (4.8) that the processes $\left(M_{\tau_{i}^{*} \wedge t}^{i}\right)_{t \geq 0}, i=1,2$, are uniformly integrable martingales. Therefore, taking the expectations in (4.8) and letting $n$ go to infinity, we apply the Lebesgue dominated convergence theorem to obtain the equalities

$$
E_{s}\left[e^{r \tau_{i}^{*}} G_{i}\left(S_{\tau_{i}^{*}}\right)\right]=E_{s}\left[e^{r \tau_{i}^{*}} V_{i}\left(S_{\tau_{i}^{*}}\right)\right]=V_{i}(s)
$$

for all $a_{*}<s<b^{\prime}$ and $a^{\prime}<s<b_{*}$, and every $i=1,2$. The latter, together with the inequalities in (4.7), implies the fact that $V_{i}(s)$ coincide with the value functions $V_{i}^{*}(s), i=1,2$, from (2.3).

Acknowledgments. The author is grateful to an anonymous Referee for their careful reading of the manuscript and helpful suggestions which allowed to improve the presentation of the paper. This research was supported by a Small Grant from the Suntory and Toyota International Centres for Economics and Related Disciplines (STICERD) at the London School of Economics and Political Science.

\section{References}

[1] Alvarez, L. H. R. (2001). Reward functionals, salvage values, and optimal stopping. Mathematical Methods of Operations Research 54 (315-337). 
[2] Alvarez, L. H. R. (2008). A class of solvable stopping games. Applied Mathematics and Optimization 49 (265-295).

[3] Battauz, A., De Donno, M. and Sbuelz, A. (2012). Real options with a double continuation region. Quantitative Finance 12(3) (465-475).

[4] Battauz, A., De Donno, M. and Sbuelz, A. (2014). Real options and American derivatives: the double continuation region. Management Science 61(5) (1094-1107).

[5] Beibel, M. and Lerche, H. R. (1997). A new look at optimal stopping problems related to mathematical finance. Statistica Sinica 7 (93-108).

[6] Beibel, M. and Lerche, H. R. (2000). A note on optimal stopping of regular diffusions under random discounting. Theory of Probability and Its Applications 45 (657$669)$.

[7] Bensoussan, A. and Lions, J. L. (1982). Applications of Variational Inequalities in Stochastic Control. North Holland, Amsterdam.

[8] Borodin, A. N. and Salminen, P. (2002). Handbook of Brownian Motion. (Second Edition) Birkhäuser, Basel.

[9] Dayanik, S. and Karatzas, I. (2003). On the optimal stopping problem for onedimensional diffusions. Stochastic Processes and Their Applications 107 (173-212).

[10] Detemple, J. (2006). American-Style Derivatives: Valuation and Computation. Chapman and Hall/CRC, Boca Raton.

[11] De Donno, M., Palmowski, Z. and Tumilewicz, J. (2018). Double continuation regions for American and swing options with negative discount rate in Lévy models. Preprint (24 pp).

[12] Dynkin, E. B. (1963). The optimum choice of the instant for stopping a Markov process. Soviet Mathematical Doklady 4 (627-629).

[13] Fakeev, A. G. (1970). Optimal stopping rules for stochastic processes with continuous parameter. Theory of Probability and Its Applications 15 (324-331).

[14] Gapeev, P. V. and Lerche, H. R. (2011). On the structure of discounted optimal stopping problems for one-dimensional diffusions. Stochastics: An International Journal of Probability and Stochastic Processes 83 (537-554).

[15] Karatzas, I. and Shreve, S. E. (1991). Brownian Motion and Stochastic Calculus. (Second Edition) Springer, New York.

[16] Lamberton, D. and Zervos, M. (2006). On the problem of optimally stopping a one-dimensional Itô diffusion. Preprint.

[17] Liptser, R. S. and Shiryaev, A. N. (2001). Statistics of Random Processes I. (Second Edition, First Edition 1977) Springer, Berlin. 
[18] Mucci, A. G. (1978). Existence and explicit determination of optimal stopping times. Stochastic Processes and Their Applications 8 (33-58).

[19] Øksendal, B. (1998). Stochastic Differential Equations. An Introduction with Applications. (Fifth Edition) Springer, Berlin.

[20] Øksendal, B. and Reikvam, K. (1998). Viscosity solutions of optimal stopping problems. Stochastics and Stochastics Reports 62 (285-301).

[21] Peskir, G. (2007). A change-of-variable formula with local time on surfaces. Séminaire de Probababilité XL. Lecture Notes in Mathematics 1899, Springer (69-96).

[22] Peskir, G. and Shiryaev, A. N. (2006). Optimal Stopping and Free-Boundary Problems. Birkhäuser, Basel.

[23] Revuz, D. and Yor, M. (1999). Continuous Martingales and Brownian Motion. (Third Edition) Springer, Berlin.

[24] Rogers, L. C. G. and Williams, D. (1987). Diffusions, Markov Processes and Martingales II. Itô Calculus. Wiley, New York.

[25] Salminen, P. (1985). Optimal stopping of one-dimensional diffusions. Mathematische Nachrichten 124 (85-101).

[26] Shepp, L. A. and Shiryaev, A. N. (1996). A dual Russian option for selling short. In Probability Theory and Mathematical Statistics: Lectures presented at the semester held in St. Peterburg, Russia, March 2 April 23, 1993. Ibragimov, I. A. et al. eds. Gordon and Breach, Amsterdam (209-218).

[27] Shiryaev, A. N. (1999). Essentials of Stochastic Finance. World Scientific, Singapore.

[28] XiA, J. and Zhou, X. (2007). Stock loans. Mathematical Finance 17 (307-317). 